\title{
Towards a Novel Framework for the Assessment of Enterprise Application Integration Packages
}

\author{
Marinos Themistocleous ${ }^{1}$, Marinos.Themistocleous@brunel.ac.uk \\ Zahir Irani, Zahir.Irani@brunel.ac.uk \\ Information Systems Evaluation and Integration Network Group (ISEing) \\ Department of Information Systems and Computing, Brunel University, UK
}

\begin{abstract}
In addressing enterprise integration problems, a diversity of technologies such as CORBA and XML were promoted, yet no single integration technology solves all integration problems. As a result, a new generation of software called Enterprise Application Integration (EAI) is emerging to addresses many integration problems by combining a diversity of integration technologies (e.g. message brokers, adapters, XML). Since EAI is a new research area, there is an absence of literature discussing issues like its adoption, evaluation and implementation. This paper, examines the application of two frameworks for the evaluation of EAI packages in the practical arena. In doing so, the authors use case study strategy to investigate integration issues. Empirical data derived from the case study suggest additions to the two evaluation frameworks. Therefore, the authors revised and extend previous works by proposing a novel evaluation framework for the assessment of EAI packages. The proposed framework makes novel contribution at two levels. First, at the conceptual level, as it incorporates criteria identified separately in previous studies as evaluation criteria. The proposed framework can be used as a decision-making tool and, supports management when taking decisions regarding the adoption of EAI. Additionally, it can be used by researchers to analyse and understand the capabilities of EAI packages.
\end{abstract}

Keywords: EAI, ERP, Evaluation,

\section{Introduction}

During the 1990s, enterprise resource planning technology came along as an approach to integration problem (Davenport, 1998). ERP systems support generic processes and are integrated packages that automate core business activities such as finance, human resources, manufacturing and supply and distribution (Gibson et al., 1999; Holland and Light,
1999). As a result, organisations attempt to parameterise ERP packages to better support their business processes and strategies. However, customisation is a difficult task that causes significant integration problems as ERP systems are: (a) complex; (b) non-flexible and, (c) often not designed to collaborate with other autonomous applications (Sprott, 2000; Stefanou, 2000). Thus, it appears that ERP systems have failed to achieve applications integration and coexist alongside other systems (Kalakota and Robinson, 2001; Themistocleous et al., 2001).

In recent years, an emerging generation of integration software called Enterprise Application Integration (EAI) has attempted to effectively address many integration problems. EAI incorporates functionality from a diversity of systems and results in the development of flexible, and maintainable integrated IT infrastructures. As a result, organisations are turning to EAI to achieve intra and inter-organisational integration. Most types (e.g. custom, ebusiness) and all combinations of information systems (e.g. custom-to-packaged-toebusiness) can be pieced together using EAI, with Themistocleous (2002) classifying all possible combinations of systems being integrated.

EAI software is based on a set of more than 16 integration technologies such as message brokers, adapters, XML etc. to piece together applications. Each EAI package is based on a subset of these 16 technologies and thus, the quality and the performance of EAI software differs form EAI package to package. The reason for this is that each EAI vendor configures its EAI software using a different subset of integration technologies. Therefore, organisations need to evaluate EAI
$1 \quad$ Corresponding Author: E-mail:
Marinos Themistocleous

Marinos.Themistocleous@brunel.ac.uk 
packages to select an appropriate one. However, enterprise application integration is a new research area with limited literature regarding the evaluation of EAI software. In addressing this void, this paper investigates the evaluation of EAI software. In doing so, the authors adopt two evaluation frameworks proposed in normative literature for EAI software evaluation. Then, they compare and use these frameworks for assessing EAI packages. The authors employ an interpretative, qualitative case study approach to investigate the evaluation of EAI software. The paper then discusses the empirical evidences derived from the case study with the authors proposing modifications to the evaluation frameworks.

\section{Integration Technologies and Enterprise Application Integration Packages}

Enterprise application integration technology incorporates multiple intra and inter-organisational applications with a central maintainable and flexible integration infrastructure. This infrastructure is responsible for the co-ordination of all integration tasks. Numerous approaches were proposed in the normative literature to describe EAI technology. Duke et al.(1999) among others suggest that a solution based on EAI involves the transportation and transformation of information between one or more applications using an integrated infrastructure. Duke et al.(1999) also suggest that EAI supports:

- the timing and sequencing rules that govern when the transportation and transformation takes place and,

- the integrity constrains that determine the success or failure of the integration.

In investigating more this approach, Themistocleous (2002) proposes that EAI achieves applications integration through four layers namely:

- connectivity;

- transportation;

- translation and

- process automation.

In a typical EAI scenario, applications elements like data and objects are extracted from a source system through the connectivity layer. Data elements are transferred to the central integrated infrastructure using transportation layer. Then, the integration infrastructure, using the translation layer, translates and reformats these elements in a valid format for target application(s). Thereafter, the process integration layer automates business processes, routes application elements to the target system(s) and triggers new events. An EAI integration infrastructure is based (in most cases) on noninvasive technologies (e.g. message brokers). The use of non-invasive technologies results in minimum changes to target and source applications. Thus, if a system requires changes only this system and its connections (connectivity layer) to the central infrastructure are altered. Therefore, EAI solutions result in maintainable and flexible infrastructures since, changes to interconnected systems are minimised. In addition, EAI offers many benefits to organisations such as (a) reduces operational costs; (b) increases productivity; (c) improves planning in supply chain management and, (d) increases collaboration among trading partners (Themistocleous and Irani 2001).

However, the adoption of EAI is widely prohibited by the confusing nature of integration technologies (Duke et al., 1999; Ring and Ward-Dutton, 1999). A diversity of integration technologies such as message brokers, adapters, CORBA and XML exist in the marketplace with each technology claiming to solve integration problems (Themistocleous and Irani, 2002). As a result, there is much technological confusion surrounding the capabilities of each technology. Ring and Ward-Dutton (1999) among others, support that no integration technology solves all types of integration problems, as each technology was designed to address a broad category of integration issues (e.g. message integration, objects integration). Hence, a combination of integration technologies is required to achieve enterprise and cross enterprise integration. Linthicum (1999) and Ruh et al.(2000) classify integration technologies into:

- database oriented middleware (e.g. ODBC, JDBC);

- message oriented technologies (e.g. message brokers, MOM, RPC, XML);

- object oriented technologies (e.g. CORBA, COM, DCOM, EJB, etc.);

- transaction based technologies (e.g. transaction process monitors, applications servers) and, 
- interface oriented technologies (e.g. adapters, application programming interfaces [APIs], screen wrappers)

EAI vendors have realised the capabilities of integration technologies and configured their EAI packages using a subset of integration technologies. The explanation for using a subset and not all technologies is attributed to many technologies overlapping in functionality. For instance CORBA, COM, DCOM and EJB support object(s) integration (Zahavi, 1999). Therefore, EAI vendors use one or two of these technologies when configuring an EAI package based on the integration problem that their package solves (e.g. an EAI package may focus on legacy integration which means that CORBA is more appropriate in this case since, it supports custom built environments). Initially EAI vendors developed tools (e.g. IBM MQSeries) based on these integrated technologies. For example EAI vendors such as Mercator have developed an integration broker and its own adapters using message brokers' and adapters' technology, respectively. Mercator has put together its integration broker, adapters and other integration software from third parties to develop an EAI package. Thus, Mercator used integration technologies to develop its EAI package. However, Mercator's EAI package differs in functionality and performance from other EAI packages since, it is based on a unique configuration of integration technologies and tools.

The complexity of integration technologies and packages have resulted in confusion. Nonetheless, organisations and researchers need to understand and evaluate the capabilities of each integration technology and EAI package. Hence, there is a need for two evaluation frameworks. The first framework should focus on the evaluation of integration technologies whereas the second on the assessment of EAI packages themselves. In addressing the first issue, Themistocleous (2002) and Themistocleous and Irani (2002) proposed and empirically validated the use of a novel framework for the evaluation of integration technologies. Likewise, Ring and WardDutton (1999) and Puschmann and Alt (2001) have also proposed frameworks for the evaluation of EAI packages. However, what remains under investigation is to empirically validate the proposed frameworks.

The authors have adopted the evaluation frameworks proposed by Ring and Ward-Dutton (1999) and Puschmann and Alt (2001) for the assessment of EAI packages. Themistocleous (2002) investigated more these two frameworks and concluded that although these frameworks were developed independently they are based on similar evaluation criteria. Thus, the authors summarise both evaluation frameworks in Table 1.

Table 1 shows that both Puschmann and Alt (2001) and Ring and Ward-Dutton (1999) assess the capabilities of EAI packages to support the:

- integration of system types (intraorganisational [custom, packaged] and interorganisational [e-business]);

- type of integration (loose, tight) (see Puschmann and Alt (2001)) and,

- availability of EAI packages that can be configured individually or used as a toolkit by users. This criterion indicates that EAI packages consist of a set of tools that are based on integration technologies. This criterion tests whether EAI packages allow integrators to customise these technologiestools (e.g. adapters) based on their own needs. 


\begin{tabular}{|c|c|c|}
\hline \multicolumn{2}{|c|}{ Evaluation Criteria proposed by } & \multirow[b]{2}{*}{ Description } \\
\hline $\begin{array}{l}\text { Puschmann } \\
\text { and Alt (2001) }\end{array}$ & $\begin{array}{l}\text { Ring and Ward- } \\
\text { Dutton (1999) }\end{array}$ & \\
\hline $\begin{array}{l}\text { Integrated } \\
\text { Toolkit }\end{array}$ & $\begin{array}{l}\text { Application } \\
\text { Toolkit }\end{array}$ & $\begin{array}{l}\text { Toolkit criterion describes whether an EAI package can be used as an out- } \\
\text { of-box product. In this case integrators have no understanding about the } \\
\text { technical details of the package but they just know what the package does. } \\
\text { Although toolkit packages ideally refer to total EAI solutions there is no } \\
\text { such solution available today (Ring and Ward-Dutton, 1999). Application or } \\
\text { Integrated EAI packages are tool-based solutions, which are accessible by } \\
\text { integrators who can use the tools to upgrade and enhance the system. }\end{array}$ \\
\hline Tightly Vs Loosely & Tight Vs Loose & $\begin{array}{l}\text { Tight and loose criteria refer to the connectivity mechanism that EAI } \\
\text { packages support. In most cases organisations require both types of } \\
\text { mechanisms with loose integration related to asynchronous communication } \\
\text { and tight to synchronous communication (Serain, 1999). }\end{array}$ \\
\hline $\begin{array}{l}\text { Individual } \\
\text { Standard EAI }\end{array}$ & $\begin{array}{l}\text { Custom }{ }_{\text {Packaged EAI }} \\
\text { Pa }\end{array}$ & $\begin{array}{l}\text { This set of criteria tests whether an EAI package focuses on the integration } \\
\text { of custom (individual) systems or packaged (standard) applications. }\end{array}$ \\
\hline $\begin{array}{l}\text { Intra } \quad \text { VS Inter- } \\
\text { organisational EAI }\end{array}$ & $\begin{array}{ll}\text { Internal } & V s \\
\text { External EAI }\end{array}$ & $\begin{array}{l}\text { Likewise, internal and external EAI criteria examine whether an EAI } \\
\text { package achieves the integration of processes and systems at enterprise and } \\
\text { cross-enterprise level. In most cases, organisations require both types of } \\
\text { integration. }\end{array}$ \\
\hline
\end{tabular}

Table 1: Evaluation Criteria for the Assessment of EAI Packages derived from the frameworks proposed by Puschmann and Alt (2001) and Ring and Ward-Dutton (1999)

\section{Research Methodology}

The aim of this paper is to investigate the evaluation of EAI packages. In doing so, the paper adopts the evaluation criteria proposed by Ring and WardDutton (1999) and Puschmann and Alt (2001) for the assessment of EAI software.

The authors have selected an interpretive, qualitative case study approach to conduct this research. An interpretitivism approach was adopted for this research since, the aim of this paper is to understand how organisations evaluate EAI packages. An interpretivism stance allows the authors to navigate and explain this phenomenon. Also, the authors suggests that in the context of this research a qualitative approach is more appropriate as such approaches can be used to: (a) investigate little-known phenomena like EAI packages evaluation; (b) examine in depth complex processes (EAI software assessment); (c) examine the phenomenon in its natural setting and, (d) learn from practice. In addition, the authors use a case study strategy to explore and understand the evaluation of EAI software. In doing so, various data collection methods such as interviews, documentation, and observation were used. The bias that is considered to be a danger in using qualitative research approach is overcome in this research by data triangulation. The use of multiple data collection methods makes the triangulation possible which provides stronger substation of theory (Eisenhardt, 1989). For the purpose of this paper, three types of triangulation are used namely: (a) data (Denzin, 1978); (b) methodological and, (c) interdisciplinary triangulation (Janesick, 2000) were used.

\section{Case Data}

Due to confidentiality reasons, the authors use the name AUTOCORP to refer to the organisation being reported. AUTOCORP is a multinational organisation that traditionally operates in the automotive sector. It has up to 200.000 employees in 132 countries and has an annual turnover of $€ 31.6$ billions. The organisation consists of 250 subsidiaries and affiliated companies in 50 countries. AUTOCORP has 185 production plants worldwide, with 43 of them located in its home-country with the rest remaining in Europe, Africa, Asia, Australia and 
North and South America. AUTOCORP also holds interest in 37 joint-venture companies around the globe. AUTOCORP is not only a name for automotive equipment such as driver information systems, ABS, brakes, and fuel-injection technology but also, for a whole range of further product areas. Examples are household appliances, automation technology, power tools, communications technology, thermo-technology, and packaging machinery. These worldwide activities of AUTOCORP are divided into four business unitssectors namely: (a) automotive equipment; (b) communication technology; (c) consumer goods and, (d) capital goods.

Recently the changes in the business environment have led AUTOCORP to considered new ways of gaining competitive advantage. A major problem that holds AUTOCORP to increase its performance or to work closer with its trading partners was the non-integrated nature of its IT infrastructure. For that reason, the organisation took the decision to address this problem by developing an integrated IT infrastructure based on an EAI solution. Many other factors has influenced AUTOCORP in making the decision for EAI adoption including among others: (a) EAI benefits; (b) EAI barriers; (c) EAI costs; (d) the technological solution that EAI supports; (e) increased competition and, (f) strategic factors (e.g. gaining competitive advantage).

AUTOCORP has decided to implement an integrated IT infrastructure at a global level. In doing so, the organisation will incorporate all types of information systems (custom, package and ebusiness applications) by integrating all permutations of system types (e.g. custom-topackaged, custom-to-ebusiness) using EAI packages. The global EAI project was started in December 2000 and will have a 6-year duration. AUTOCORP estimates that by December 2006 the organisation will be able to function worldwide in an integrated way.

Before proceeding to the implementation of EAI project, the IT department of AUTOCORP took the decision to evaluate integration technologies and products. As reported by project manager, AUTOCORP believes that there is no single EAI product that supports all integration requirements of its IT architecture. To decide which of the available
EAI products is suitable for the AUTOCORP business bus (the proposed integration infrastructure for AUTOCORP), the company evaluated five different EAI products from vendors like: (a) BEA Systems; (b) CrossWorlds; (c) IBM; (d) Level 8 Systems and, (e) Mercator Software. Among others, the evaluation process focuses on the following criteria:

- Integrated Vs Toolkit application: With the implementation of SAP R/3, AUTOCORP needs to integrate several mainframe and custom-built applications, for which no standard adapters can be used. Additionally, AUTOCORP already uses IBMs MQ Series for the physical transport of data between applications. Therefore, the EAI product must be like a toolkit application, which: (a) allows the developers to individually build adapters for in-house developed systems and, (b) can be used with existing tools that are already used as a standard for application integration.

- Tightly Vs Loosely coupling: The connectivity services of an EAI product enable data integration by using synchronous and asynchronous mechanisms. By applying one of these mechanisms, it can be differentiated if the EAI solution supports a more tightly or a more loosely coupling of applications. An integrator reported that EAI products that support a tightly coupled application assist companies with synchronous integration, whereas EAI solutions that support loose coupling assist companies with asynchronous integration. Similarly to the majority of other organisations that have adopted EAI solutions, both synchronous and asynchronous mechanisms are applied in the case of AUTOCORP. A prominent example for asynchronous data integration is the exchange of master and transactional data between distributed ERP systems. In contrast, synchronous mechanisms are very often used for e-business applications, which support available-to-promise checks in the ERP systems. As AUTOCORP needs a flexible architecture that supports both asynchronous and synchronous integration 
scenarios, the company needs an EAI product that supports both.

- Individual Vs Standard application integration: A major component of EAI products are the interface services that provide functionality for the translation of different application's APIs and object models. Most of the EAI vendors, such as CrossWorlds and Level8 Systems, have concentrated on APIs and object models of standard business applications like SAP R/3, Oracle and Baan. Only few vendors like BEA or Mercator Software that originally built traditional middleware solutions deliver EAI products that support the customer with functionality for the integration of legacy systems such as IBM CICS or Siemens BS2000. Especially for historical grown, multinational companies like AUTOCORP that have already used legacy systems, the support of such systems is crucial as the replacement of them is often not profitable. The reason for this is that many of these legacy systems: (a) are reliable; (b) handle critical applications [in the case of AUTOCORP] (e.g. manufacturing systems); (c) the replacement of these systems will be risky and cost high amounts of money; (d) their functionality is difficult to be replaced by other systems, since they are specialised and, (e) there is no justification, time and money for their replacement.

- Intra Vs Inter-organisational integration: The integration of intra and interorganisational business processes and applications was another evaluation criteria for AUTOCORP. The organisation requires an EAI product that supports the integration of both intra and inter-organisational applications and processes. The evaluation of EAI products indicates that EAI vendors with strong middleware background such as BEA Systems and Level 8 Systems support intra-organisational EAI. The relatively new vendor-players in the EAI market like CrossWorlds have specialised in providing e-business integration and therefore, support more inter-organisational EAI. CrossWorlds offers standard e-commerce process configurations, which can be customized easily with a graphical workflow-modelling tool. Mercator Software has a background in the integration of EDI and back end systems. This is the explanation for Mercator policy to integrate a wide range of EDI standards and scenarios such as UN/EDIFACT and Odette in its Mercator product suite.

Apart from the evaluation criteria presented in Table 1, AUTOCORP used many other criteria. However, the organisation was reluctant to share these criteria for confidentiality reasons. When the project manager was asked to comment he reported that:

"The confusing nature in the
integration marketplace requires
employees with EAI skills and
integration technologies. Currently,
there is a shortage of skilled
employees... our company has spent
money and time to acquire this
knowledge. We believe that enterprises
that have this knowledge [IT
sophistication on EAI] are in a position
to adopt EAI. The sooner you adopt this
knowledge the better."

It appears that AUTOCORP had difficulties in understanding integration technologies due to the confusion in the integration marketplace. It seems that an evaluation framework supported the organisation to adopt EAI, since it improved IT sophistication and allowed AUTOCORP to understand the capabilities of integration technologies and EAI packages. When the authors asked the project manager to report only a few of these evaluation criteria that influence the decision for selecting an appropriate EAI package he reported:

"We all know that integration is achieved at different integration layers. Each EAI package uses its own set of tools or technologies at each level. So it is important for us to clarify two issues: (a) which technologies and tools are used by an EAI package and, (b) what 


\section{integration layer does these technologies cover..."}

To investigate more this issue, the authors asked three integrators, three designers and four consultants (internal and external) to identify the most important evaluation criteria. Nearly all of them (nine out of ten) agreed with the project manager and mentioned that evaluation criteria that focus on integration layers help them to assess EAI packages. Based on interviewee's comments it appears that the integration layers proposed by Themistocleous (2002) namely: (a) connectivity; (b) transportation; (c) translation and, (d) process automation can be used as evaluation criteria. This is also supported by empirical evidences reported by Themistocleous (2002).

\section{Data Analysis and Synthesis}

Empirical evidence confirms the literature findings (Ring and Ward-Dutton, 1999; Linthicum, 2000a; 2001; Puschmann and Alt, 2001) that EAI packages consist of a set of integration technologies with each EAI vendor customising its own package. This means that EAI vendors configure their products using integration technologies (e.g. adapters) to support a specific market (e.g. custom-to-packaged applications integration). For instance, one vendor may use adapters to support packaged-to-packaged integration, where another may use adapters to support custom-to-packaged applications integration. This implies that organisations may need a framework to evaluate EAI packages, and understand their capabilities before proceeding to the adoption of a solution.

The empirical data presented in section 4 have shown that eight criteria were used by AUTOCORP for the evaluation of EAI packages. These criteria confirm literature findings (Ring and Ward-Dutton, 1999; Puschmann and Alt, 2001). Moreover, the authors expand the frameworks proposed by Ring and Ward-Dutton, (1999) and Puschmann and Alt (2001) by adding the integration layers as evaluation criteria. Integration layers are criteria proposed by Themistocleous (2002) for the assessment of integration technologies. The decision for incorporating integration layers with the evaluation framework was not based only on the empirical evidences reported in this paper. The decision was also made since there exist other case studies that suggest that integration layers can be used as evaluation criteria when evaluating integration technologies and EAI packages (Themistocleous 2002).

The revised evaluation framework allows decisionmakers to understand which integration layers an EAI package supports as well as to realise the integration technologies that are used. The latter allows decision-makers to refer to the framework for evaluating integration technologies proposed by Themistocleous (2002), and assess these technologies. This imposes a correlation between the two evaluation frameworks. In addition, decisionmakers can evaluate EAI packages using the eight criteria identified by AUTOCORP to further clarify the differences among EAI packages. Table 2 presents the revised framework for the evaluation of EAI packages. The framework contributes to the selection of appropriate EAI packages. It also improves IT sophistication since it contributes to understanding the capabilities of EAI packages.

Based on the evaluation results, AUTOCORP took the decision to adopt a variety of EAI products to integrate within the organisation. This proves that none of the EAI packages evaluated meets all evaluation criteria set by the organisation. Considering that the five EAI packages assessed represent the elite of EAI solutions (Ring and WardDutton, 1999) it appears that there is no single EAI package that addresses all integration problems. This finding is in accordance of other literature findings (Linthicum, 2000b; Ruh et al., 2000; Linthicum, 2001). For that reason, the IBM MQ Series integrator was used for the messages brokering. BEA elink was selected to support tightly and loosely coupling and Mercator software to facilitate inter and intra-organisational application integration. In addition, all three products can be used as toolkit applications and individual EAI. For confidentiality reasons, AUTOCORP members refused to provide more information regarding the applicability (range and level of use) of each product in the organisation. 


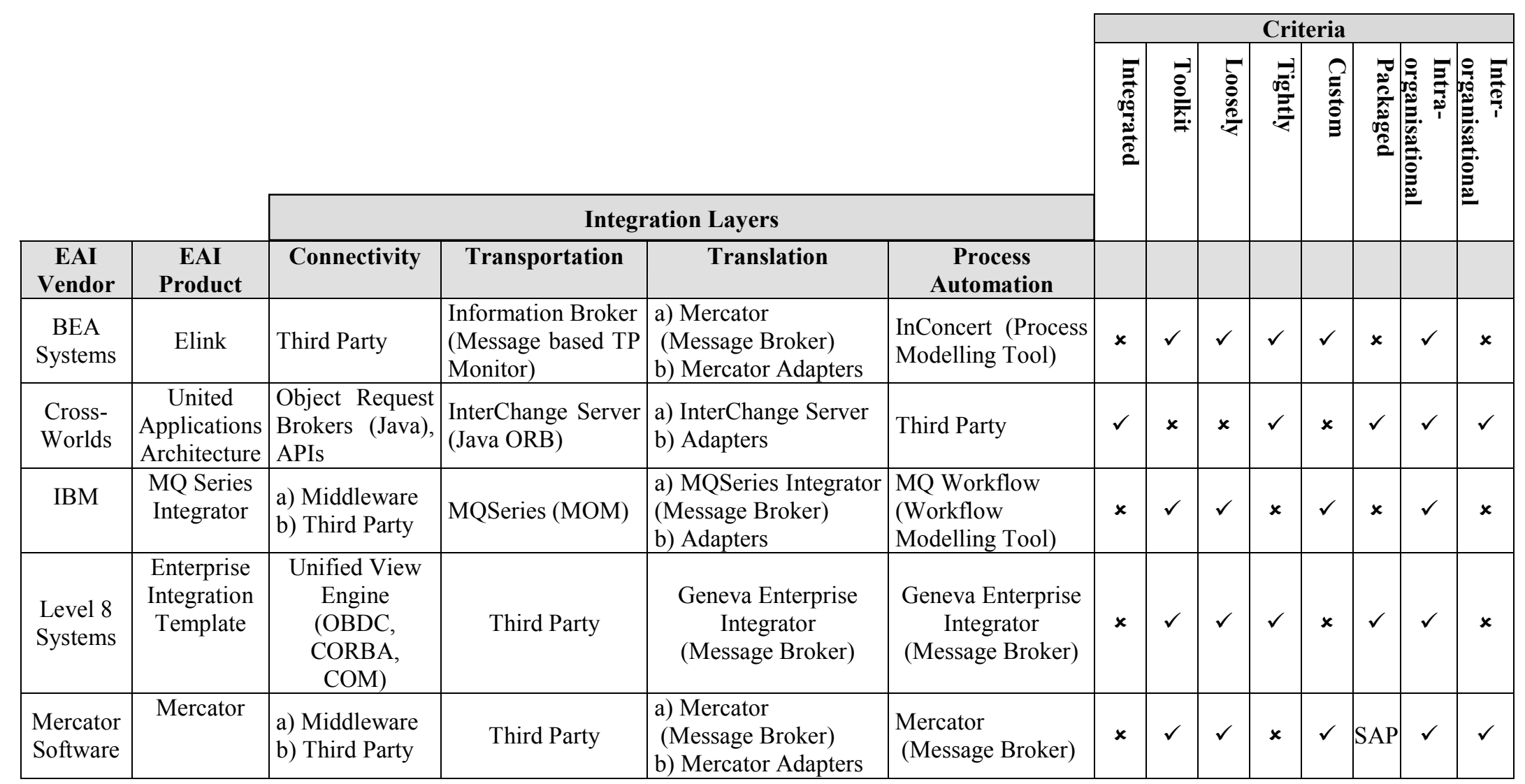

Table 2: Novel Framework for Evaluating EAI Packages 


\section{Conclusions}

The rapidly changing nature of business environments requires organisations to be more flexible to gain competitive advantage. However, the non-integrated nature of existing IT infrastructures prohibits organisations to achieve their targets. In overcoming this problem, organisations are turning into a new generation of software called enterprise application integration to fully integrate their applications and business processes. The adoption of EAI supports business goals as it results in many business benefits such as return on investment and achieves a flexible and manageable IT infrastructure.

Before adopting an EAI solution, organisations need to evaluate EAI packages and integration technologies. The reason for this is that there is marketplace confusion and a variety of tools and technologies that support systems integration. In addition, there is no single EAI package or integration technology that addresses all integration problems. Therefore, organisations need to understand the nature of each technology and EAI package before taking their decision to adopt integration technologies. Since EAI is a new research area there is limited literature regarding evaluating EAI packages and integration technologies. A literature review shows that there are two available frameworks for the evaluation of EAI packages. However, these frameworks have not been validated using empirical data. For that reason, the authors have adopted these frameworks and investigated their applicability through testing them empirically. In doing so, the authors conducted a case study in a multinational organisation.

Empirical evidences suggest taking into consideration the four integration layers when evaluating integration technologies. As a result, the authors develop a novel framework that includes both the four integration layers and the eight criteria that derived from the two evaluation frameworks. Thus, the novel framework for the evaluation of EAI packages includes twelve evaluation criteria that focus on:

- Connectivity layer;

- Transportation layer;

- Translation layer;

- Process automation layer;

- Integrated product;

- Toolkit product;

- Loosely type of integration;

- Tightly type of integration;

- Custom systems integration;

- Packaged systems integration;

- Intra-organisational integration and

- Inter-organisational integration.

The proposed novel framework can be used for decision-making when organisations take decisions regarding EAI package adoption. Since, the authors can not generalise the data derived from a single case study, they suggest that the proposed framework will allow others to relate their experiences to those reported.

\section{References}

Davenport, T. 1998. 'Putting the Enterprise into the Enterprise System', Harvard Business Review, July-August 1998): 121-131.

Denzin, N. Y. K. 1978. 'The Research Act: A Theoretical Introduction to Sociological Methods', McGraw Hill, New York, USA,

Duke, S., Makey, P. and Kiras, N. 1999. 'Application Integration Management Guide: Strategies and Technologies', Butler Group Limited, Hull, UK.

Eisenhardt, K. M. 1989. 'Building Theories from Case Study Research', Academy of Management Review, 14(4): 532-550.

Gibson, N., Holland, C. and Light, B. 1999. 'Enterprise Resource Planning: A Business Approach to Systems Development', Proceedings of 32nd Hawaii International Conference on System Sciences, Hawaii, USA., [CD Proceedings].

Holland, C. and Light, B. 1999. 'Global Enterprise Resource Planning Implementation', Proceedings of 32nd Hawaii International Conference on System Sciences, Hawaii, USA, [CD Proceedings]. 
Janesick, V. 2000. 'The choreography of qualitative research design' In Handbook of qualitative research (Ed, Lincoln, Y. S.) Sage publications, Thousand Oaks, CA, 379-399.

Kalakota, R. and Robinson, M. 2001. 'e-Business 2.0: Roadmap for Success', AddisonWesley, Boston, Massachusetts, USA, 0201-72165-1.

Linthicum, D. 2000a. 'B2B Application Integration', Addison-Wesley, Massachusetts, USA, 0-201-70936-8.

Linthicum, D. 1999. 'Enterprise Application Integration', Addison-Wesley, Massachusetts, USA, 0-201-64583-5.

Linthicum, D. 2000b. 'Mercator: eBusiness Integration Software in an SAP Environment', Mercator Software, Wilton, Connecticut, USA.

Linthicum, D. 2001. 'Mercator: Next Generation Application Integration', Mercator Software, Wilton, Connecticut, USA.

Puschmann, T. and Alt, R. 2001. 'Enterprise Application Integration - The Case of the Robert Bosch Group', Proceedings of the 34th Hawaii International Conference on System Sciences, Maui, Hawai, USA, [CD Proceedings].

Ring, K. and Ward-Dutton, N. 1999. 'Enterprise Application Integration: Making the Right Connections', Ovum Ltd, London, UK, 1-902566-15-7.

Ruh, W., Maginnis, F. and Brown, W. 2000. 'Enterprise Application Integration: A Wiley Tech Brief', Wiley John Wiley \& Sons Inc., New York, USA, 0-47137641-8.

Serain, D. 1999. 'Middleware', Winder, R., Practitioner Series, Springer, Berlin, Germany, 1-85233-011-2.

Sprott, D. 2000. 'Componentizing the Enterprise Application Packages', Communications of the ACM, 43(4): 63-69.

Stefanou, C. 2000. 'The Selection Process of Enterprise Resource Planning (ERP) Systems', Proceedings of 2000 Americas Conference on Information Systems, AMCIS 2000, Long Beach, California, USA, pp. 988-991.

Themistocleous, M. 2002. 'Evaluating the Adoption of Enterprise Application Integration in Multinational Organisations', PhD Thesis, Department of Information Systems and Computing, Brunel University, London.
Themistocleous, M. and Irani, Z. 2002. 'Evaluating Enterprise Application Integration Technologies: A Novel Frame of References', European Journal of Operational Research, In Press

Themistocleous, M. and Irani, Z. 2001. 'Benchmarking the Benefits and Barriers of Application Integration', Benchmarking: An International Journal, 8(4): 317-331.

Themistocleous, M., Irani, Z. and O'Keefe, R. 2001. 'ERP and Application Integration: Exploratory Survey', Business Process Management Journal, 7(3): 195-204.

Zahavi, R. 1999. 'Enterprise Application Integration with CORBA', John Wiley and Sons Inc, New York, USA, 0471327204. 